\title{
Cognitive Non-ideal NOMA Satellite-Terrestrial Networks with Channel and Hardware Imperfections
}

\author{
Yerassyl Akhmetkaziyev, Galymzhan Nauryzbayev, ${ }^{\circ}$ Sultangali Arzykulov, ${ }^{\circ}$ Ahmed M. Eltawil, \\ and * Khaled M. Rabie \\ School of Engineering and Digital Sciences, Nazarbayev University, Nur-Sultan, Z05H0K3, Kazakhstan \\ ${ }^{\circ}$ Computer, Electrical, and Mathematical Sciences \& Engineering Division, KAUST, Thuwal, KSA 23955-6900 \\ *Department of Engineering, Manchester Metropolitan University, Manchester, M15 6BH, UK \\ Email: \{yerassyl.akhmetkaziyev, galymzhan.nauryzbayev\}@nu.edu.kz, \\ ○\{sultangali.arzykulov@gmail.com, ahmed.eltawil@kaust.edu.sa\}, *k.rabie@mmu.ac.uk
}

\begin{abstract}
This paper investigates a non-orthogonal multiple access (NOMA) assisted cognitive satellite-terrestrial network which is practically limited by interference noises, transceiver hardware impairments, imperfect successive interference cancellation, and channel state information mismatch. Generalized outage probability expressions for NOMA users in both primary and secondary networks are derived considering the impact of interference temperature constraint. Finally, obtained results are corroborated by Monte Carlo simulations and compared with the orthogonal multiple access to show the superior performance of the proposed network model.
\end{abstract}

Index Terms-Cognitive radio (CR), non-orthogonal multiple access (NOMA), outage probability, satellite-terrestrial network (STN).

\section{INTRODUCTION}

Satellite-terrestrial networks (STNs) have received significant attention from research and industry due to their capability of providing a connection to rural and distant areas, where the deployment of terrestrial infrastructure is uneconomical/difficult. Moreover, the STNs are considered as a promising enabler of future fifth-generation $(5 \mathrm{G})$ networks that expect a 1000fold data traffic increase [1]. This leads to a critical issue of spectrum scarcity [2]. It can be resolved by deploying cognitive radio $(\mathrm{CR})$ in the STNs to enable spectrum sharing between the satellite and terrestrial networks [3].

Another criteria for the successful implementation of $5 \mathrm{G}$ is massive connectivity. Traditionally, satellite-terrestrial systems principally exploit an orthogonal multiple access (OMA) scheme to deliver services; however, it is considered as one of the bottlenecks since there is a limitation in the number of servable devices due to their orthogonality. Therefore, the non-orthogonal multiple access (NOMA) technique has been recently proposed as a promising technology for the future wireless networks because of its ability to maintain resource sharing (i.e., time, code and frequency) and, at the same time, differentiate the end-users with respect to different power levels [4]-[8]. In [6]-[8], the underlay CR and NOMA were jointly studied aiming at reducing the interference and more effective spectrum utilization. For example, in [6], the authors evaluated the outage performance of CR-NOMA by considering an amplify-and-forward relaying scheme while a similar system model was examined for the detect-and-forward scheme over generalized $\alpha-\mu$ fading channels [7], [8].

Until now, very few papers studied the performance of NOMA in cognitive STNs [9]-[11]. For instance, the authors in [9] derived the closed-form outage probability (OP) and approximated ergodic capacity expressions for the primary and secondary users for a decode-and-forward relaying protocol. In [10], the authors examined the NOMA-assisted overlay multiuser cognitive STN and revealed its superiority over traditional benchmark schemes. Furthermore, in [11], the authors derived the analytical expression using Meijer-G functions for the ergodic capacity of NOMA-assisted cognitive STNs and confirmed its advantages.

In contrast to [9]-[11], this paper examines the cognitive NOMA-based STN considering the aggregate transceiver distortions, channel and successive interference cancellation (SIC) imperfections, hardware impairment (HI) as well as interference temperature constraint (ITC). Hence, the key contributions of this paper are as follows. First, closed-form analytical OP expressions are derived for both primary and secondary users in the considered cognitive NOMA-assisted STN. Second, the influence of several system parameters on the network performance is investigated using derived analytical results, and, by comparing the NOMA users' OP performance with a benchmark OMA scheme, the advantage of NOMA is verified. Finally, the correctness of derived analytical findings are validated by the Monte Carlo simulations and, therefore, the correctness of analytical results are verified.

\section{System Model}

Consider a downlink underlay multi-user CR-assisted STN model consisting of secondary and primary networks as illustrated in Fig. 1. The primary network $(\mathrm{PN})$ includes the satellite transmitter $(T)$ which intends to directly communicate with multiple primary users (PUs), indicated by $R_{n}, n \in$ $\{1,2, \ldots, N\}$. At the same time, the secondary network (SN) consists of a base station $(S)$, which can access the licensed band spectrum of PUs and communicate with the secondary users (SUs), denoted by $U_{k}, k \in\{1,2, \ldots, K\}$. In this case, the PUs can suffer from the aggregate interference from $S$ while $T$ will interfere with the SUs. 


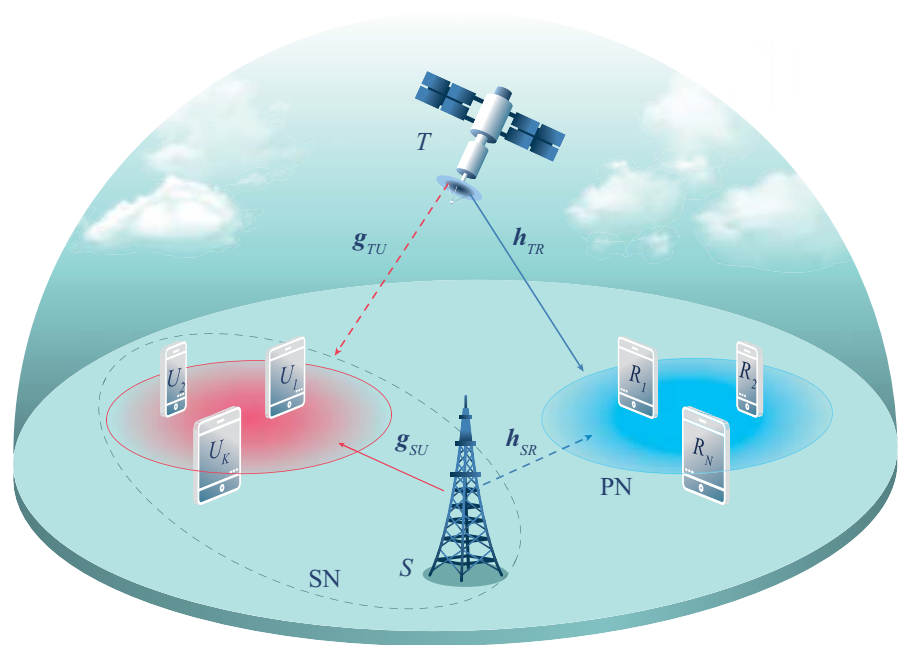

Fig. 1. Cognitive NOMA-assisted STN model.

\section{A. Channel Models}

We model the communication links using linear minimum mean square error channel estimator as [12] $\chi=\tilde{\chi}+\epsilon$, where $\chi$ denotes the observed mismatched channel, while $\tilde{\chi}$ is a real channel estimate and $\epsilon$ indicates its estimation error, with $\mathcal{C N}(0, \lambda)$, where $\lambda$ can be described considering the nominal transmit SNR $\omega$, as $\lambda=\Phi \omega^{-\eta}$ [13]. The perfect CSI can be obtained if $\eta \rightarrow \infty$ for $\omega>0$. Moreover, the additive white Gaussian noise (AWGN), with mean zero and variance $\sigma^{2}$, exposes all the receiving nodes.

1) Satellite Direct and Interference Channels: The channels corresponding to the satellite links given by $\mathbf{g}_{T U}=$ $\left[g_{T 1}, g_{T 2}, \ldots, g_{T K}\right]$ and $\mathbf{h}_{T R}=\left[h_{T 1}, h_{T 2}, \ldots, h_{T N}\right]$ follow shadowed-Rician fading. Thus, the corresponding probability density function (PDF) is given by [14]

$$
f_{\left|\chi_{n}\right|^{2}}(x)=\sum_{\ell=0}^{m_{n}-1} \Upsilon x^{\ell} e^{-\partial_{n} x}, x \geq 0,
$$

with $\Upsilon=\frac{1}{2 \bar{b}_{n}}\left(\frac{2 \bar{b}_{n} m_{n}}{2 \bar{b}_{n} m_{n}+\Omega_{n}}\right)^{m_{n}} \frac{\left(1-m_{n}\right)_{\ell}\left(-\delta_{n}\right)^{\ell}}{(\ell !)^{2}}$ and $\partial_{n}=$ $\varrho_{n}-\delta_{n}$, where $\delta_{n}=\frac{\Omega_{n}}{2 \bar{b}_{n}\left(2 \bar{b}_{n} m_{n}+\Omega_{n}\right)}, \varrho_{n}=\frac{1}{2 \bar{b}_{n}}$. Here, $2 \bar{b}_{n}$ is the average power of a multi-path component, $\Omega_{n}$ denotes the average power of a line-of-sight (LoS) component, and $m_{n}$ represents the Nakagami parameter. Furthermore, as the Friis' law for free-space propagation states, the pathloss for satellite communication links has linear dependence with logarithmic distance as in [15] and can be expressed by $L(D)=\frac{1}{\mathcal{K}_{\mathcal{B}} \mathcal{T} \mathcal{W}}\left(\frac{c}{4 \pi f_{c} D}\right)^{2}$, where $D$ represents the distance between $T$ and the user of interest ${ }^{1}$, while $c$ and $f_{c}$ denote the light speed and carrier frequency, respectively. $\frac{1}{\mathcal{K}_{\mathcal{B}} \mathcal{T} \mathcal{W}}$ is a scale factor, where $\mathcal{K}_{\mathcal{B}}, \mathcal{T}$ and $\mathcal{W}$ denote the Boltzmann's constant, receiver noise temperature and carrier bandwidth, respectively.

We denote the antenna gain at $T$ as $G_{T}$, with $\varphi$ representing the angle between the $i$-th user's location and the beam center

\footnotetext{
${ }^{1}$ Without loss of generality, it is assumed that the distances between the satellite and terrestrial users are equal.
}

with respect to the satellite. Hence, the beam gain $G_{i}(\varphi)$ is expressed as [16]

$$
G_{i}(\varphi)=G_{i, \max }\left(\frac{J_{1}(u)}{2 u}+36 \frac{J_{3}(u)}{u^{3}}\right)^{2},
$$

where $u=2.07123 \frac{\sin \varphi}{\sin \varphi_{3 \mathrm{~dB}}}, \varphi_{3 \mathrm{~dB}}$ is the constant $3-\mathrm{dB}$ angle for the beam and $J_{l}(\cdot)$ expresses the first-kind Bessel function of order $l$.

2) Terrestrial Direct and Interference Channels: The terrestrial links given by $\mathbf{g}_{S U}=\left[g_{S 1}, g_{S 2}, \ldots, g_{S K}\right]$ and $\mathbf{h}_{S R}=$ $\left[h_{S 1}, h_{S 2}, \ldots, h_{S N}\right]$ are presumed to abide the Nakagami$m$ fading. Therefore, the corresponding channel gains (i.e., squared values) are Gamma random variables (RVs) with the PDF given by

$$
f_{\left|\chi_{k}\right|^{2}}(y)=\frac{y^{m_{k}-1} e^{-\frac{y}{\nu}}}{\Gamma\left(m_{k}\right) \nu^{m_{k}}}
$$

where $m_{k}$ and $\nu$ stand for the shape and scale parameters.

In addition, the sectored antenna pattern has been used to model analog beamforming between communicating nodes as $G(\theta)=G_{m}$, when $\theta \leq \theta_{b}$; otherwise, $G(\theta)=G_{s}$, where $G_{m}$ and $G_{s}$ denote the main and side lobe gains ${ }^{2}$, respectively. $\theta$ is the angle of a boresight direction, while $\theta_{b}$ indicates the antenna beamwidth. For convenience, we assume that the terrestrial station interferes the PUs with side lobe gain, while a main lobe gain is exploited in the terrestrial direct links.

\section{B. Signal and SINR Models}

1) Satellite Primary Network: The satellite $T$ and $N$ terrestrial end-users $\left(R_{n, n \in\{1, \ldots, N\}}\right)$ comprise the downlink satellite PN that employs the NOMA concept by broadcasting a superposed signal $x=\sum_{n=1}^{N} \sqrt{\alpha_{n}} x_{n}$, where $x_{n}$ and $\alpha_{n}$ denote the message devoted for the $n$-th user and the corresponding power allocation (PA) coefficient (with $\alpha_{1}>\alpha_{2}>\ldots>\alpha_{N}$ s.t. $\sum_{n=1}^{N} \alpha_{n}=1$ ).

Hence, considering the CSI imperfections, the received signal at $R_{i}$, for $i \in\{1, \ldots, N\}$, can be written as

$$
\begin{aligned}
y_{i}= & \left(\tilde{h}_{i}+\epsilon_{i}\right) \sqrt{P_{T} G_{T} G_{i}(\varphi) L}\left(\sum_{n=1}^{N} \sqrt{\alpha_{n}} x_{n}+\mu_{i}\right) \\
& +h_{S i} \sqrt{P_{S} G_{S} \bar{G}_{i} d_{S i}^{-\tau}}\left(s_{k}+\bar{\mu}_{i}\right)+n_{i}
\end{aligned}
$$

where $n_{i} \sim \mathcal{C N}\left(0, \sigma_{i}^{2}\right)$ denotes the AWGN term. $\mu_{i} \sim$ $\mathcal{C N}\left(0, \kappa_{i}^{2}\right)$ and $\bar{\mu}_{i} \sim \mathcal{C N}\left(0, \bar{\kappa}_{i}^{2}\right)$ represent the impact of residual HIs by the aggregate distortion noises, where $\kappa_{i}$ and $\bar{\kappa}_{i}$ denote the compound HI levels observed in the communication links of corresponding transmitter-receiver pairs.

Then, considering imperfect SIC, the corresponding signalto-interference-noise-distortion ratio (SINDR) to decode message $x_{n}$, for $n \leqslant i$, can be written, by arranging $\rho_{i}=$

\footnotetext{
${ }^{2}$ It is assumed that an antenna gain has a constant value in the frame of given main or side lobe sectors.
} 
$P_{T} G_{T} G_{i}(\varphi) L, \bar{\rho}_{i}=P_{S} G_{S} G_{i} d_{S R i}^{-\tau}, X_{i}=\left|\tilde{h}_{T R_{i}}\right|^{2}, Y_{i}=$ $\left|h_{S R_{i}}\right|^{2}$, and $a=\alpha_{n} \rho_{i}$, as follows

$$
\gamma_{i \rightarrow n}=\frac{a X_{i}}{b X_{i}+\Sigma_{i}+c Y_{i}},
$$

where $\Sigma_{i}=\rho_{i}\left(1+k_{i}^{2}\right) \sigma_{\epsilon}^{2}+\sigma_{i}^{2}$ stands for the power of channel error and AWGN noise. The interference received from $S$ is given by $c=\left(1+\bar{\kappa}_{S R_{i}}^{2}\right) \bar{\rho}_{i} . b=\rho_{i} \mathcal{A}$ represents the SICbased interference, with $\mathcal{A}=\left(\Psi_{n}+\tilde{\Psi}_{n}+\kappa_{i}^{2}\right)$, where $\Psi_{n}=$ $\sum_{t=n+1}^{N} \alpha_{t}$, and $\tilde{\Psi}_{n}=\sum_{l=1}^{n-1} \xi_{l} \alpha_{l}$, with $0 \leq \xi \leq 1$ [17]. Note that $R_{1}$ detects its own message by regarding the other signals as noise and setting $\Psi_{1}=\sum_{t=2}^{N} \alpha_{t}$ and $\tilde{\Psi}_{1}=0$, while message $x_{N}$ is decoded only at $R_{N}$, subject to $\Psi_{N}=0$ and perfect/imperfect SIC realizations given by $\tilde{\Psi}_{n}=\sum_{l=1}^{n-1} \xi_{l} \alpha_{l}$.

2) Terrestrial Secondary Network: On the other hand, the terrestrial SN consists of the base station $S$ and $K$ NOMA end-users $\left(U_{k}, k \in\{1, \ldots, K\}\right)$, when the superimposed signal $s=$ $\sum_{k=1}^{K} \sqrt{\beta_{k}} s_{k}$ is sent to all intended SUs, where $s_{k}$ and $\beta_{k}$ indicate the message dedicated for the $k$-th $\mathrm{SU}$ and the corresponding PA coefficient (with $\beta_{1}>\beta_{2}>\ldots>\beta_{K}$ s.t. $\left.\sum_{k=1}^{K} \beta_{k}=1\right) . G_{S}$ is the antenna gain at $S$. In the same manner, the received signal at $U_{j}$ can be written as

$$
\begin{aligned}
r_{j}= & \left(\tilde{g}_{j}+\epsilon\right) \sqrt{P_{S} G_{S} G_{D_{j}} d_{j}^{-\tau}}\left(\sum_{k=1}^{K} \sqrt{\beta_{k}} s_{k}+\bar{\mu}_{i}\right) \\
& +g_{P D} \sqrt{P_{T} G_{T} G_{D}(\varphi) L}\left(x_{n}+\mu_{j}\right)+w_{j},
\end{aligned}
$$

Hence, the SINDR of $U_{j}$ to detect message $s_{k}$ from $S$ is as

$$
\psi_{j \rightarrow k}=\frac{W_{1} Z_{j} P_{S}}{P_{S}\left(W_{2} Z_{j}+E\right)+C Q_{j}+\sigma_{j}^{2}},
$$

where $Z_{j}=\left|\tilde{g}_{j}\right|^{2}, Q_{j}=\left|g_{T_{j}}\right|^{2}$, and $W_{1}=\beta_{k} G_{S} G_{D_{j}} d_{j}^{-\tau}$. The power of channel error and AWGN noise is denoted by $E=$ $G_{S} G_{D_{j}} d_{j}^{-\tau}\left(1+\bar{\kappa}_{i}^{2}\right) \sigma_{\epsilon}^{2} \cdot W_{2}=G_{S} G_{D_{j}} d_{j}^{-\tau} \overline{\mathcal{A}}$ stands for the SIC-based interference, with $\overline{\mathcal{A}}=\left(\Psi_{j}+\tilde{\Psi}_{j}+\bar{\kappa}_{i}^{2}\right)$, whereas $C=\left(1+\kappa_{T_{j}}^{2}\right) P_{T} G_{T} G_{D}(\varphi) L$ is the interference from $T$.

\section{Outage Probability}

\section{A. Satellite Primary Network}

By definition, the outage is defined as the probability that the SINDR value falls below a predefined signal-to-noise ratio (SNR) associated rate threshold, which can be expressed as $v=2^{\mathcal{R}}-1$, where $\mathcal{R}$ is the data rate threshold [18].

Thus, using Eq. (5), the OP of decoding message $x_{n}$ by $R_{i}$ can be determined as

$$
\mathbb{P}_{\text {out }, i}(v)=\operatorname{Pr}\left[\gamma_{i \rightarrow n}<v\right], 0<n \leq i .
$$

Proposition 1: Considering imperfect SIC/CSI and HI, we can express the closed-form expression of OP for the primary user of interest as in Eq. (9).

Proof: The full derivation is drawn in Appendix A.

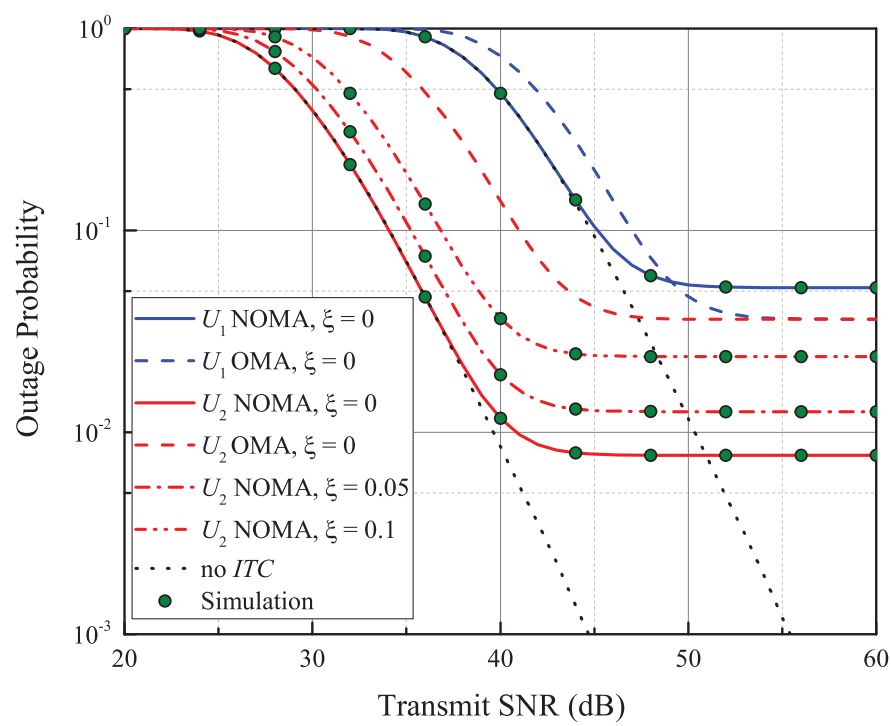

Fig. 2. The OP comparison of the NOMA and OMA for the SN with different $\xi=\{0,0.05,0.1\}$.

\section{B. Terrestrial Secondary Network}

The OP for $U_{K}$ can be expressed using Eq. (7) and considering ITC as in Eq. (10), where $S_{i}=\frac{I_{\mathrm{ITC}}^{[i]}}{Y_{i}}$ and $P_{S}=$ $\min \left(\bar{P}_{S}, S^{*}\right)$ by assuming $S^{*}=\min \left(S_{1}, S_{2}, \ldots, S_{N}\right)$. Further, we denote $I_{\mathrm{ITC}}^{[i]}=I_{\mathrm{ITC}} d_{S R_{i}}^{\tau}$ and $Y_{i}=\left|h_{S R_{i}}\right|^{2}$.

For the convenience, we simplify the expression by using the following definitions: $T_{1}=\frac{v\left(E P_{s}+\sigma_{j}^{2}\right)}{P_{s}\left(W_{1}-v W_{2}\right)}, T_{2}=$ $\frac{v C}{P_{s}\left(W_{1}-v W_{2}\right)}, \quad R_{1}=\frac{v \sigma_{j}^{2}}{I_{\mathrm{ITC}}^{[i]}\left(W_{1}-v W_{2}\right)}, R_{2}=\frac{v C}{I_{\mathrm{ITC}}^{[i]}\left(W_{1}-v W_{2}\right)}$, $R_{3}=\frac{v I_{I T C}^{[i]} E}{I_{\mathrm{ITS}}^{[i]}\left(W_{1}-v W_{2}\right)}$, and $\Lambda=\frac{I_{\mathrm{ITC}}^{[i]}}{P_{s}}$.

Proposition 2: The OP in the SN given by Eq. (10) can be expressed in closed form considering the ITC as sum of Eqs. (12) and (11), where, for the sake of brevity, the terms $A$ and $B$ are demonstrated separately.

$$
\begin{array}{r}
A=\left(\sum_{\ell=0}^{m_{n}-1} \Upsilon \ell ! \partial_{n}^{-\ell-1}-e^{\frac{-T_{1}}{\nu_{0}}} M_{A} T_{2}^{n} \sum_{\ell=0}^{m_{n}-1} \Upsilon(n+\ell) !\right. \\
\left.\left(\frac{T_{2}}{\nu_{0}}+\partial_{n}\right)^{-n-\ell-1}\right) \frac{\gamma\left(m_{k}, \frac{\Lambda}{\nu}\right)}{\Gamma\left(m_{k}\right)} .
\end{array}
$$

Proof: The full derivation can be found in Appendix B.

\section{Numerical Results}

This section provides the numerical results on the outage performance for the system model under consideration designated by the simulation framework drawn in Table I which mainly follows the parameters listed in [15], [16]. We also verify our derived analytical expressions through Monte Carlo simulations. For the sake of simplicity, we consider only two PUs and two SUs ${ }^{3}$.

\footnotetext{
${ }^{3}$ In the real-time communication, the use of multiple NOMA users may not be achievable because the processing complexity of the SIC at receivers raises in a non-linear manner by the increased number of users [21]. Moreover, when the SIC error propagates, this complexity gets to be more vital [7].
} 


$$
\begin{aligned}
\mathbb{P}_{\mathrm{out}, i}^{\mathrm{PN}}(v) & =\sum_{\ell=0}^{m_{n}-1} \frac{\Upsilon \ell !}{\partial_{n}^{\ell+1}}-\sum_{\ell=0}^{m_{n}-1} \sum_{p=0}^{\ell} \frac{\ell !}{p !} \frac{\Upsilon e^{-I(i) \Sigma_{i}\left(\partial_{n}\right)}}{\partial_{n}^{\ell-p+1}} \sum_{t=0}^{p} \frac{(I(i))^{p} p !\left(\Sigma_{i}\right)^{p-t} c^{t}}{t !(p-t) !} \frac{\Gamma\left(t+m_{k}\right)}{\Gamma\left(m_{k}\right) \nu^{m_{k}}}\left(\frac{1}{\nu}+I(i) \partial_{n} c\right)^{-t-m_{k}} \\
\mathbb{P}_{\mathrm{out}, j}^{\mathrm{SN}}(v) & =\operatorname{Pr}\left(\frac{W_{1} Z_{j} P_{S}}{P_{S}\left(W_{2} Z_{j}+E\right)+C Q_{j}+\sigma_{j}^{2}}<v, \bar{P}_{s}<S_{i}\right)+\operatorname{Pr}\left(\frac{W_{1} Z_{j} S_{i}}{S_{i}\left(W_{2} Z_{j}+E\right)+C Q_{j}+\sigma_{j}^{2}}<v, \bar{P}_{s}>S_{i}\right) \\
& =\underbrace{\operatorname{Pr}\left(Z_{j}<\left(T_{1}+T_{2} Q_{j}\right), Y_{i}<\Lambda\right)}_{A}+\underbrace{\operatorname{Pr}\left(Z_{j}<\left(R_{1} Y_{i}+R_{2} Q_{j} Y_{i}+R_{3}\right), Y_{i}>\Lambda\right)}_{B} .
\end{aligned}
$$

$$
\begin{aligned}
B=\frac{\Gamma\left(m, \frac{\Lambda}{\nu}\right)}{\Gamma(m)} \sum_{\ell=0}^{m_{n}-1} \Upsilon \ell ! \partial_{n}^{-\ell-1}-e^{\frac{-R_{3}}{\nu_{0}}} M_{B} \sum_{i=0}^{t}\left(\begin{array}{l}
t \\
i
\end{array}\right) R_{1}^{t-i} R_{2}^{i} \frac{\Gamma(t+m)}{\Gamma(m) \nu^{m}} e^{-\left(\frac{1}{\nu}+\frac{R_{1}}{\nu_{0}}\right) \Lambda} \sum_{j=0}^{t+m-1} \sum_{\ell=0}^{m_{n}-1} \frac{\Lambda^{j} \Upsilon}{j !} \\
\times \frac{V_{2} \Omega^{-(i+\ell+1)}}{\Gamma(-j+t+m)} G_{1,2}^{2,1}\left(\frac{\frac{R_{2} \Lambda}{\nu_{0}}+\partial_{n}}{\Omega} \mid \begin{array}{l}
1-(i+\ell+1) \\
0,-(i+\ell+1)+(-j+t+m)
\end{array}\right)
\end{aligned}
$$

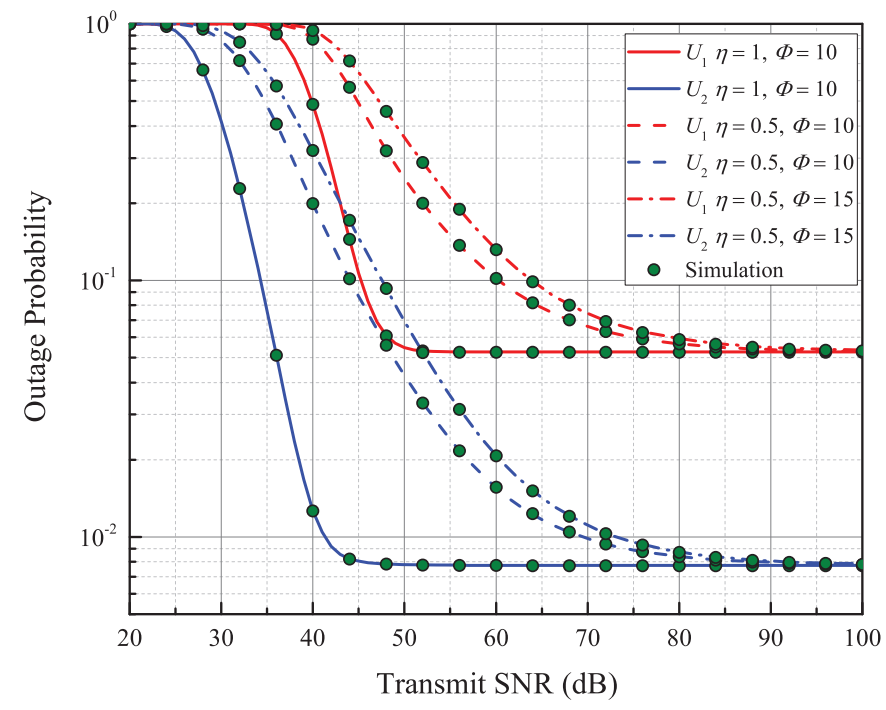

(a) The OP performance for the SN with perfect CSI and SNR-independent CSI mismatches $\{0,0.05\}$ and $\{0,0.1\}$.

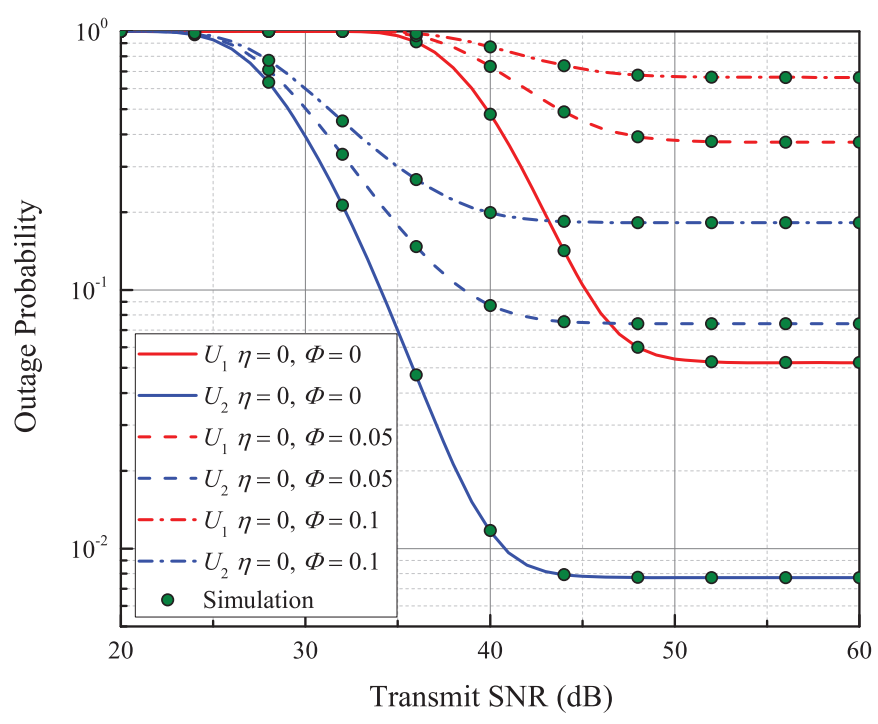

(b) The OP performance for the SN with perfect CSI and SNR-dependent CSI mismatches $\{1,10\},\{0.5,10\}$ and $\{0.5,15\}$.

Fig. 3. The OP versus the transmit SNR for the terrestrial SN for different CSI scenarios.

Table I. Simulation parameters.

\begin{tabular}{|l|c|}
\hline Parameter & Value \\
\hline \hline Terrestrial channel parameter, $m_{0}$ & 2 \\
Satellite channel parameters, $\left\{m_{i}, b_{i}, \Omega_{i}\right\}$ & $\{5,0.251,0.279\}$ \\
Terrestrial antenna gains, $\left\{G_{m}, G_{s}\right\}$ & $\{12,-1.1092\} \mathrm{dB}$ \\
Satellite antenna gains, $\left\{G_{T}, G_{i, \max }\right\}$ & $\{4.8,54\} \mathrm{dB}$ \\
Threshold, $\left\{\zeta_{\text {NOM }}, \zeta_{\mathrm{OMA}}\right\}$ & $\{3,12\} \mathrm{dB}$ \\
PA factors, $\left\{\alpha_{1}, \alpha_{2}\right\}$ & $\{0.7,0.3\}$ \\
Path-loss exponent, $\tau$ & 2 \\
Orbit height, $D$ & $35786 \mathrm{~km}$ \\
$S$-to- $R_{N}$ distance, $\left\{d_{R_{1}}, d_{R_{2}}\right\}$ & $\{150,75\} \mathrm{m}$ \\
$S$-to- $U_{K}$ distance, $\left\{d_{U_{1}}, d_{U_{2}}\right\}$ & $\{100,50\} \mathrm{m}$ \\
$3 \mathrm{~dB}$ angle, $\varphi_{3 d B}$ & $0.4^{\circ}$ \\
Carrier frequency, $f_{c}$ & $2 \mathrm{GHz}$ \\
Interference noise power & $10 \mathrm{~dB}$ \\
Temperature, $\mathcal{T}$ & $300 \mathrm{~K}$ \\
Carrier bandwidth, $\mathcal{W}$ & $15 \mathrm{MHz}$ \\
\hline
\end{tabular}

In Fig. 2, the OP comparison of the analytical results for a NOMA network with the simulated results for a OMA scheme is demonstrated for both SUs with $I T C=10 \mathrm{~dB}$. Here, to ensure comparison fairness, the data rate requirements of OMA are set as two-fold for NOMA, and transmit power at $S$ is set to $0.5 P$. In addition, the asymptotic case with no ITC, i.e., ITC $\rightarrow \infty$, is plotted and it demonstrates the best performance without saturation. Moreover, it is clearly seen that the NOMA-based network outperforms the OMA in terms of OP. However, one can observe the outage of $U_{1}$ in the NOMAassisted network starts saturating at lower levels of the transmit SNR compared to the OMA network. It can be explained by the $I T C$ equation, where the transmit power of $0.5 P_{s}$ rises the values of the $I T C$ at $S$; therefore, the level of transmit SNR, where the OP curves start saturating, increases. Additionally, the influence of various SIC scenarios is investigated for $U_{2}$ 
only, since $U_{1}$ is independent of SIC. According to the results, SIC-enabled end-user $U_{2}$ performs better than $U_{1}$ within the system model. Although, subject to the flawed SIC, the outage of $U_{2}$ deteriorates when $\xi$ increases, as expected. It is worth mentioning that, at lower levels of the transmit SNR, imperfect SIC has a low impact on the performance.

Fig. 3 shows the impact of CSI mismatch for two users in the terrestrial SN. Moreover, it considers the SNRindependent/dependent CSI scenarios. When $\eta=0$, meaning that the channel error variance is independent of the transmit SNR, it is observed that $\Phi$ significantly affects the system performance. Noticeably, $\Phi$ does not have any effect on the OP performance at low SNR values. Particularly, it starts influencing $U_{1}$ at $35 \mathrm{~dB}$ and $U_{2}$ at $25 \mathrm{~dB}$, approximately. It happens due to the fact that when $\Phi$ approaches 0 , the CSI quality tends to become perfect. Therefore, the small mismatch in CSI does not affect the performance at low SNR. Contrarily, when $\eta \neq 0$, CSI becomes SNR-dependent and achieves saturation only at a greater rate of the transmit SNR in comparison with the SNR-independent CSI. From Fig. 3(b), one can clearly observe that the increase of $\eta$ leads to the improvement of system performance. Similarly, in the case of equal $\eta$ values, the growth of $\Phi$ also results in a slight improvement of the OP performance. However, it is apparent that $\eta$ has much more influence on the performance than $\Phi$. It is worth mentioning that, despite the overall system improvement, all curves level out on the same OP rate in the high-SNR region.

\section{Conclusion}

In this paper, we have evaluated the performance of cognitive NOMA-assisted STNs wherein terrestrial SUs enjoy the access to spectrum with the satellite PN limited only by interference temperature constraint. Different from existing works, we have applied NOMA to both primary and secondary networks and obtained the closed-form OP expressions taking into account the hardware, SIC and CSI imperfections along with the interference noises. Particularly, we have shown the deteriorative effects of imperfect CSI and SIC. Furthermore, a comparison with the OMA network, which is considered as a benchmark scheme, revealed that the proposed NOMA-based STN demonstrates outstanding performance advancement while utilizing the spectrum resource in an efficient manner. Finally, derived analytical findings were verified by Monte Carlo simulations.

\section{ACKNOWLEDGMENT}

This work was supported by the Nazarbayev University Faculty Development Competitive Research Program under Grant no. 240919FD3935.

\section{APPENDIX A \\ SATELLITE PRIMARY NETWORK}

In this section, we present the derivation steps for appraising the outage metric of primary NOMA users.
Hence, the OP can be evaluated using Eq. (5) as

$$
\begin{aligned}
& \mathbb{P}_{\text {out }, i}(v)=\operatorname{Pr}\left[X_{i} \leq I(i)\left(\Sigma_{i}+c Y_{i}\right)\right] \\
&=\int_{0}^{\infty} \underbrace{\left(\int_{0}^{I(i)\left(\Sigma_{i}+c Y_{i}\right)} f_{X_{i}}(x) \mathrm{d} x\right)}_{A_{1}} f_{Y}(y) \mathrm{d} y,
\end{aligned}
$$

where $I(i)=\frac{v}{a_{i}-b_{i} v}$. Here $A_{1}$ can be solved by integrating Eq. (1) with the aid of [19, Eq. (3.351.1)] as

$$
\begin{aligned}
A_{1} & =\int_{0}^{I(i)\left(\Sigma_{i}+c Y_{i}\right)} \sum_{\ell=0}^{m_{n}-1} \Upsilon x^{\ell} e^{-\partial_{n} x} \mathrm{~d} x=\sum_{\ell=0}^{m_{n}-1} \Upsilon\left(\frac{\ell !}{\partial_{n}^{\ell+1}}\right. \\
& \left.-e^{-I(i)\left(\Sigma_{i}+c Y_{i}\right) \partial_{n}} \times \sum_{p=0}^{\ell} \frac{\ell !}{p !} \frac{\left(I(i)\left(\Sigma_{i}+c Y_{i}\right)\right)^{p}}{\partial_{n}^{\ell-p+1}}\right) .
\end{aligned}
$$

Further, by denoting $\Delta=I(i)\left(\Sigma_{i}+c Y_{i}\right), \quad \Delta^{p}$ can be expanded using a binomial theorem as $\Delta^{p}=$ $(I(i))^{p} \sum_{t=0}^{p}\left(\begin{array}{l}p \\ t\end{array}\right)\left(\Sigma_{i}\right)^{p-t}\left(c Y_{i}\right)^{t}$. Now, for the terrestrial interference link, the i.n.i.d. Gamma RVs with shape $m_{k}$ and scale $\nu$ parameters are generated using the PDF in Eq. (3). Substituting Eq. (A.2) into Eq. (A.1) and by using $\Delta^{p}$, the outage can be rewritten as

$$
\begin{aligned}
\mathbb{P}_{\text {out }, i}(v)= & \sum_{\ell=0}^{m_{n}-1} \Upsilon \frac{\ell !}{\partial_{n}^{\ell+1}} \int_{0}^{\infty} \frac{y^{m_{k}-1} e^{-\frac{y}{\nu}}}{\Gamma\left(m_{k}\right) \nu^{m_{k}}} \mathrm{~d} y \\
& -\sum_{\ell=0}^{m_{n}-1} \sum_{p=0}^{\ell} \frac{\ell !}{p !} \frac{\Upsilon e^{-I(i) \Sigma_{i} \partial_{n}}}{\partial_{n}^{\ell-p+1}}(I(i))^{p} \sum_{t=0}^{p}\left(\begin{array}{c}
p \\
t
\end{array}\right) \\
\times & \frac{\left(\Sigma_{i}\right)^{p-t} c^{t}}{\Gamma\left(m_{k}\right) \nu^{m_{k}}} \int_{0}^{\infty}\left(y_{i}\right)^{t+m_{k}-1} e^{-y\left(\frac{1}{\nu}+I(i) \partial_{n} c\right)} \mathrm{d} y .
\end{aligned}
$$

Finally, we can express the exact OP with the aid of [19, Eq. (8.310.1)] in its closed form as Eq. (9).

\section{APPENDIX B}

\section{TERRESTRIAL SECONDARY NETWORK}

The term $A$ in (10) can be rewritten as

$$
A=\underbrace{\int_{q=0}^{\infty} f_{Q}(q) \underbrace{\int_{z=0}^{T_{1}+T_{2} Q_{j}} f_{Z}(z) \mathrm{d} z}_{A_{1}} z}_{A_{2}} \mathrm{~d} q \underbrace{\int_{y=0}^{\Lambda} f_{Y}(y) \mathrm{d} y}_{A_{3}}
$$

The PDFs of $Z$ and $Y$ abide by Nakagami- $m$ statistical model and $Q$ term follow Shadowed-Rician distribution. Therefore, by using the PDF in Eq. (3), we obtain $A_{1}=1-e^{\frac{-T_{1}-T_{2} Q_{j}}{\nu_{0}}} M_{A}\left(T_{2} Q_{j}\right)^{n}$, where $M_{A}=$ $\sum_{p=0}^{m_{0}-1} \frac{1}{\nu_{0}^{p} \Gamma(p+1)} \sum_{n=0}^{p}\left(\begin{array}{l}p \\ n\end{array}\right) T_{1}^{p-n}$. Now, using $A_{1}$, the PDF in Eq. (1) and [19, Eq. (3.351.3)], we can calculate $A_{2}$ as

$$
A_{2}=\left(1-e^{\frac{-T_{1}-T_{2} Q_{j}}{\nu_{0}}} M_{A}\left(T_{2} Q_{j}\right)^{n}\right) \sum_{\ell=0}^{m_{n}-1} \Upsilon \int_{0}^{\infty} q^{\ell} e^{-\partial_{n} q} \mathrm{~d} q
$$




$$
\begin{aligned}
& =\sum_{\ell=0}^{m_{n}-1} \Upsilon\left(\int_{0}^{\infty} q^{\ell} e^{-\partial_{n} q} \mathrm{~d} q-e^{\frac{-T_{1}}{\nu_{0}}} M_{A} T_{2}^{n} \int_{0}^{\infty} q^{n} q^{\ell} e^{-\left(\frac{T_{2}}{\nu_{0}}+\partial_{n}\right) q} \mathrm{~d} q\right) \\
& =\sum_{\ell=0}^{m_{n}-1} \Upsilon\left(\ell ! \partial_{n}^{-\ell-1}-e^{\frac{-T_{1}}{\nu_{0}}} M_{A} T_{2}^{n}(n+\ell) !\left(\frac{T_{2}}{\nu_{0}}+\partial_{n}\right)^{-n-\ell-1}\right)
\end{aligned}
$$

and the CDF of $A_{3}$ can be obtained independently as $\frac{\gamma\left(m_{k}, \frac{\Lambda}{\nu}\right)}{\Gamma\left(m_{k}\right)}$. Finally, by inserting $A_{2}$ and $A_{3}$ into Eq. (B.1), we express the term $A$ in closed-form as Eq. (12).

The term $B$ in Eq. (10) can be determined as

$$
B=\int_{q=0}^{\infty} f_{Q}(q) \underbrace{\int_{y=\Lambda}^{\infty} f_{Y}(y) \int_{0}^{R_{1} Y+R_{2} Y Q_{j}+R_{3}} f_{Z}(z) \mathrm{d} z \mathrm{~d} y}_{B_{1}} \mathrm{~d} q .
$$

Here, using Eq. (3), $B_{1}$ can be calculated as

$$
\begin{aligned}
& B_{1}=\left(1-e^{\frac{-R_{1} Y-R_{2} Y Q_{j}-R_{3}}{\nu_{0}}} \sum_{p=0}^{m_{0}-1} \frac{\left(R_{3}+Y\left(R_{1}+R_{2} Q_{j}\right)\right)^{p}}{\nu_{0}^{p} \Gamma(p+1)}\right) \\
& \times \int_{\Lambda}^{\infty} \frac{y^{m-1} e^{-\frac{y}{\nu}}}{\Gamma(m) \nu^{m}} \mathrm{~d} y=\frac{\Gamma\left(m, \frac{\Lambda}{\nu}\right)}{\Gamma(m)}-e^{\frac{-R_{3}}{\nu_{0}}} M_{B}\left(R_{1}+R_{2} Q_{j}\right)^{t} \\
& \times \int_{\Lambda}^{\infty} \frac{y^{t} y^{m-1} e^{-y\left(\frac{1}{\nu}+V_{1} Q_{j}\right)}}{\Gamma(m) \nu^{m}} \mathrm{~d} y=\frac{\Gamma\left(m, \frac{\Lambda}{\nu}\right)}{\Gamma(m)}-e^{\frac{-R_{3}}{\nu_{0}}} M_{B} \\
& \times \underbrace{\left(R_{1}+R_{2} Q_{j}\right)^{t} \frac{\Gamma\left(t+m,\left(\frac{1+\nu V_{1} Q_{j}}{\nu}\right) \Lambda\right)}{\Gamma(m) \nu^{m}}\left(\frac{1+\nu V_{1} Q_{j}}{\nu}\right)^{-t-m}}_{B_{2}},
\end{aligned}
$$

where $M_{B}=\sum_{p=0}^{m_{0}-1} \frac{1}{\nu_{0}^{p} \Gamma(p+1)} \sum_{t=0}^{p}\left(\begin{array}{l}p \\ t\end{array}\right)\left(R_{3}\right)^{p-t}, V_{1}=\frac{R_{1}+R_{2}}{\nu_{0}}$.

Now, we need to apply the series representation of the upper incomplete Gamma function given by [19] as $\Gamma(b, c)=$ $\Gamma(b) e^{-c} \sum_{i=0}^{b-1} \frac{c^{i}}{i !}$ and binomial theorem in order to expand $B_{2}$ in Eq. (B.4). Then, by using the aforementioned methods and after some mathematical manipulations, we rewrite $B_{2}$ as

$$
\begin{aligned}
B_{2}= & \sum_{i=0}^{t}\left(\begin{array}{l}
t \\
i
\end{array}\right) R_{1}^{t-i}\left(R_{2} Q_{j}\right)^{i} \sum_{j=0}^{t+m-1} \frac{\Gamma(t+m) \Lambda^{j} V_{2}}{\Gamma(m) \nu^{m} j !} \\
& \times e^{-\left(\frac{1}{\nu}+\frac{R_{1}+R_{2} Q_{j}}{\nu_{0}}\right) \Lambda}\left(1+\Omega Q_{j}\right)^{j-t-m},
\end{aligned}
$$

where $V_{2}=\left(\frac{\nu_{0}+\nu R_{1}}{\nu_{0} \nu}\right)^{j-t-m}$ and $\Omega=\frac{\nu R_{2}}{\nu_{0}+\nu R_{1}}$. Thus, the term $B$ can be rewritten as

$$
\begin{aligned}
& B=\frac{\Gamma\left(m, \frac{\Lambda}{\nu}\right)}{\Gamma(m)} \sum_{\ell=0}^{m_{n}-1} \Upsilon \int_{0}^{\infty} q^{\ell} e^{-\partial_{n} q} \mathrm{~d} q-e^{\frac{-R_{3}}{\nu_{0}}} M_{B} \sum_{i=0}^{t}\left(\begin{array}{l}
t \\
i
\end{array}\right) \\
& \times R_{1}^{t-i} R_{2}^{i} \frac{\Gamma(t+m)}{\Gamma(m) \nu^{m}} e^{-\left(\frac{1}{\nu}+\frac{R_{1}}{\nu_{0}}\right) \Lambda} \sum_{j=0}^{t+m-1} \frac{\Lambda^{j}}{j !} V_{2} \sum_{\ell=0}^{m_{n}-1} \Upsilon \\
& \times \int_{0}^{\infty}\left(1+\Omega Q_{j}\right)^{-(t+m-j)} q^{i+\ell+1-1} e^{-\left(\frac{R_{2} \Lambda}{\nu_{0}}+\partial_{n}\right) q} \mathrm{~d} q .
\end{aligned}
$$

Finally, by using the Meijer-G function represented with the aid of [20, Eqs. (7.34.3.46.1) and (7.34.3.271.1)], where $e^{-b \gamma}=$
$G_{0,1}^{1,0}\left(\left.b \gamma\right|_{0} ^{-}\right)$and $(1+c \gamma)^{-d}=\frac{1}{\Gamma(d)} G_{1,1}^{1,1}\left(\left.c \gamma\right|_{0} ^{1-d}\right)$, and after some mathematical manipulations with the additional aid of [19, Eq. (3.351.3)], we obtain the closed-form expression of term $B$ as in Eq. (11).

\section{REFERENCES}

[1] L. Dai et al., "A Survey of Non-Orthogonal Multiple Access for 5G," IEEE Commun. Surveys Tuts., vol. 20, no. 3, pp. 2294-2323, 3rd quarter 2018.

[2] G. Giambene, S. Kota, and P. Pillai, "Satellite-5G Integration: A Network Perspective," IEEE Netw., vol. 32, no. 5, pp. 25-31, Oct. 2018.

[3] Y. Ruan et al., "Spectral-Energy Efficiency Tradeoff in Cognitive Satellite-Vehicular Networks Towards Beyond 5G," IEEE Wireless Commun. Netw. Conf. (WCNC), Marrakesh, Morocco, pp. 1-6, 2019.

[4] M. Liu, T. Song, and G. Gui, "Deep Cognitive Perspective: Resource Allocation for NOMA-Based Heterogeneous IoT With Imperfect SIC," IEEE Internet Things J., vol. 6, no. 2, pp. 2885-2894, Apr. 2019.

[5] S. Arzykulov et al., "Underlay Spectrum Sharing for NOMA Relaying Networks: Outage Analysis," Int. Conf. Comput., Netw. Commun. (ICNC), Big Island, HI, USA, 2020, pp. 897-901.

[6] S. Arzykulov et al., "Performance Analysis of Underlay Cognitive Radio Nonorthogonal Multiple Access Networks," IEEE Trans. Veh. Technol., vol. 68, no. 9, pp. 9318-9322, Sept. 2019.

[7] S. Arzykulov et al., "Hardware- and Interference-Limited Cognitive IoT Relaying NOMA Networks With Imperfect SIC Over Generalized NonHomogeneous Fading Channels," IEEE Access, vol. 8, pp. 72942-72956, 2020.

[8] G. Nauryzbayev et al., "Underlay CR-NOMA Relaying Networks over Non-Homogeneous Generalized Fading Channels," 12th Int. Symp. Commun. Syst., Netw. Digit. Signal Process. (CSNDSP), Porto, 2020, pp. 1-6.

[9] X. Zhang et al., "Performance Analysis of NOMA-Based Cooperative Spectrum Sharing in Hybrid Satellite-Terrestrial Networks," IEEE Access, vol. 7, pp. 172321-172329, 2019.

[10] V. Singh, P. K. Upadhyay, and M. Lin, "On the Performance of NOMAAssisted Overlay Multiuser Cognitive Satellite-Terrestrial Networks," IEEE Wireless Commun. Lett., vol. 9, no. 5, pp. 638-642, May 2020.

[11] X. Yan et al., "On the ergodic capacity of NOMA-based cognitive hybrid satellite terrestrial networks," 2017 IEEE/CIC Int. Conf. Commun. China (ICCC), Qingdao, 2017, pp. 1-5.

[12] G. Nauryzbayev and E. Alsusa, "Interference Alignment Cancellation in Compounded MIMO Broadcast Channels With General Message Sets," IEEE Trans. Commun., vol. 63, no. 10, pp. 3702-3712, Oct. 2015.

[13] G. Nauryzbayev, E. Alsusa, and M. Abdallah, "On the Feasibility of Interference Alignment in Compounded MIMO Broadcast Channels With Antenna Correlation and Mixed User Classes," IEEE Trans. Veh. Technol., vol. 67, no. 3, pp. 2130-2140, Mar. 2018.

[14] A. Abdi et al., "A new simple model for land mobile satellite channels: first- and second-order statistics," IEEE Trans. Wireless Commun., vol. 2, no. 3, pp. 519-528, May 2003.

[15] K. Guo et al., "Outage analysis of cognitive hybrid satellite-terrestrial networks with hardware impairments and multi-primary users," IEEE Wireless Commun. Lett., vol. 7, no. 5, pp. 816-819, Oct. 2018.

[16] P. K. Sharma, D. Deepthi, and D. I. Kim, "Outage Probability of 3-D Mobile UAV Relaying for Hybrid Satellite-Terrestrial Networks," IEEE Commun. Lett., vol. 24, no. 2, pp. 418-422, Feb. 2020.

[17] X. Li et al., "Residual Transceiver Hardware Impairments on Cooperative NOMA Networks," IEEE Trans. Wireless Commun., vol. 19, no. 1, pp. 680-695, Jan. 2020

[18] G. Nauryzbayev, M. Abdallah, and K. M. Rabie, "Outage Probability of the EH-Based Full-Duplex AF and DF Relaying Systems in $\alpha-\mu$ Environment," IEEE Veh. Technol. Conf. Fall, USA, 2018, pp. 1-6.

[19] I. S. Gradshteyn and I. M. Ryzhik, Table of integrals, series, and products. Elsevier/Academic Press, Amsterdam, 7th ed., 2007.

[20] Wolfram, "The wolfram functions site." Last visited on 03/10/2020

[21] Z. Ding, M. Peng, and H. V. Poor, "Cooperative non-orthogonal multiple access in 5G systems," IEEE Commun. Lett., vol. 19, pp. 1462-1465, Aug. 2015. 